\title{
Multiplicity of positive solutions for Kirchhoff type problem involving critical exponent and sign-changing weight functions
}

\section{Chang-Mu Chu*}

\section{"Correspondence:}

gzmychuchangmu@sina.com

College of Science, Guizhou Minzu

University, Guiyang, Guizhou

550025, People's Republic of China

\begin{abstract}
This paper is devoted to the study of a class of Kirchhoff type problems with critical exponent, concave nonlinearity, and sign-changing weight functions. By means of variational methods, the multiplicity of the positive solutions to this problem is obtained.
\end{abstract}

MSC: 35J20; 35J60; 47J30; 58E50

Keywords: Kirchhoff type problem; critical exponent; concave nonlinearity; sign-changing weight functions; variational methods

\section{Introduction and main results}

This paper is concerned with the existence and multiplicity of positive solutions for the following problem:

$$
\begin{cases}-\left(a+b \int_{\Omega}|\nabla u|^{2} d x\right) \Delta u=\lambda f(x) u^{q}+g(x) u^{5}, & \text { in } \Omega, \\ u=0, & \text { on } \partial \Omega,\end{cases}
$$

where $\Omega$ is a smooth bounded domain in $R^{3}$ with $0<q<1$ and the parameters $a, b, \lambda>0$. The weight functions $f(x), g(x)$ satisfy the following conditions:

$\left(f_{1}\right) f(x) \in L^{q_{0}}(\Omega)$ and $f^{+}=\max \{f, 0\} \neq 0$, where $q_{0}=\frac{6}{5-q}$;

$\left(f_{2}\right)$ there exist positive constants $\beta_{0}$ and $\delta_{0}$ such that $B\left(x_{0}, 2 \delta_{0}\right) \subset \Omega$ and $f(x) \geq \beta_{0}$ in $B\left(x_{0}, 2 \delta_{0}\right)$

$\left(g_{1}\right) g(x) \in L^{\infty}(\Omega)$ and $g^{+}=\max \{g, 0\} \neq 0$;

$\left(g_{2}\right) g\left(x_{0}\right)=\|g\|_{\infty}$ and $g(x)>0$ for all $x \in B\left(x_{0}, 2 \delta_{0}\right)$;

$\left(g_{3}\right)$ there exists $k>3$ such that $g(x)=g\left(x_{0}\right)+o\left(\left|x-x_{0}\right|^{k}\right)$ as $x \rightarrow x_{0}$.

In (1), if we replace $\lambda f(x) u^{q}+g(x) u^{5}$ by $h(x, u)$, it reduces to the following Dirichlet problem of Kirchhoff type:

$$
\begin{cases}-\left(a+b \int_{\Omega}|\nabla u|^{2} d x\right) \Delta u=h(x, u), & \text { in } \Omega, \\ u=0, & \text { on } \partial \Omega .\end{cases}
$$

Problem (2) is related to the stationary analogue of the equation

$$
u_{t t}-\left(a+b \int_{\Omega}|\nabla u|^{2} d x\right) \Delta u=h(x, u)
$$

@2014 Chu; licensee Springer. This is an Open Access article distributed under the terms of the Creative Commons Attribution License (http://creativecommons.org/licenses/by/2.0), which permits unrestricted use, distribution, and reproduction in any medium, provided the original work is properly cited. 
proposed by Kirchhoff in [1] as an extension of the classical d'Alembert wave equation for free vibrations of elastic strings. Kirchhoff's model takes into account the changes in the length of the string produced by transverse vibrations. It received great attention only after Lions [2] proposed an abstract framework for the problem. The solvability of the Kirchhoff type problem (2) has been paid much attention to by various authors. The positive solutions of such a problem are obtained by using variational methods [3-5]. Perera and Zhang [6] obtained a nontrivial solution of problem (2) via the Yang index and the critical group. He and Zou [7] obtained infinitely many solutions by using the local minimum methods and the fountain theorems. Recently, when $h(x, u)$ is a continuous superlinear nonlinearity with critical growth, the existence of positive solutions of the Kirchhoff type problem has been studied [8-13]. Moreover, the paper [14] considered problem (2) with concave and convex nonlinearities by using a Nehari manifold and fibering map methods, and one obtained the existence of multiple positive solutions. In addition, the corresponding results of the Kirchhoff type problem can be found in [15-25], and the references therein.

In the present paper, we deal with problem (1) and consider the existence and multiplicity of positive solutions of problem (1). About the critical growth situation, the aforementioned papers only showed the existence of positive solutions of the Kirchhoff type problem. Moreover, involving the concave and convex nonlinearities, [14] only considered the subcritical growth case. Therefore, our purpose is to extend the result of [14] to critical growth. The main results of this paper extend the corresponding results in [11] and [14].

Before stating our results, we give some notations and assumptions. Let $\|w\|=$ $\left(\int_{\Omega}|\nabla w|^{2} d x\right)^{\frac{1}{2}},\|w\|_{s}=\left(\int_{\Omega}|w|^{s} d x\right)^{\frac{1}{s}}(1<s<\infty), B\left(x_{0}, \delta\right)=\left\{x \in R^{3}:\left|x-x_{0}\right|<\delta\right\}$. In addition, we denote positive constants by $C, C_{1}, C_{2}, \ldots$ The main results of this paper are as follows.

Theorem 1 Let $a>0, b>0$ and $0<q<1$. Suppose that $\left(f_{1}\right)$ and $\left(g_{1}\right)$ hold, then there exists $\Lambda>0$ such that problem (1) for all $\lambda \in(0, \Lambda)$ has at least one positive solution.

Theorem 2 Let $a>0, b>0$ and $0<q<1$. Suppose that $\left(f_{1}\right),\left(f_{2}\right),\left(g_{1}\right),\left(g_{2}\right)$ and $\left(g_{3}\right)$ hold, then there exists $\lambda^{*}>0$ such that problem (1) for all $\lambda \in\left(0, \lambda^{*}\right)$ has at least two positive solutions.

Remark 1 Our Theorem 2 extends the results for the critical case of Theorem 1.1 in [11]. Our Theorem 2 shows that we have at least two positive solutions of problem (1), but the authors of the reference only obtain at least one positive solution of problem (1). In addition, the results of Theorem 2.1 in [14] are extended to critical growth.

This paper is organized as follows. In Section 2, we give the local Palais-Smale condition. The proof of Theorems 1 and 2 is provided in Section 3.

\section{The local Palais-Smale condition}

In this section, we show that the corresponding functional of problem (1) satisfies the $(P S)_{c}$ condition. Let $u^{ \pm}=\max \{ \pm u, 0\}$, the corresponding functional of problem (1) is

$$
I(u)=\frac{a}{2}\|u\|^{2}+\frac{b}{4}\|u\|^{4}-\frac{\lambda}{q+1} \int_{\Omega} f\left(u^{+}\right)^{q+1} d x-\frac{1}{6} \int_{\Omega} g\left(u^{+}\right)^{6} d x, \quad u \in H_{0}^{1}(\Omega) .
$$


It is well known that the critical points of the functional $I$ in $H_{0}^{1}(\Omega)$ are weak solutions of problem (1). By the definition of weak solution $u$ of problem (1), it means that $u \in H_{0}^{1}(\Omega)$ satisfies

$$
\left\langle I^{\prime}(u), v\right\rangle=\left(a+b\|u\|^{2}\right) \int_{\Omega} \nabla u \cdot \nabla v d x-\lambda \int_{\Omega} f\left(u^{+}\right)^{q} v d x-\int_{\Omega} g\left(u^{+}\right)^{5} v d x
$$

for any $v \in H_{0}^{1}(\Omega)$.

Define the best Sobolev constant,

$$
S=\inf _{u \in H_{0}^{1}(\Omega) \backslash\{0\}} \frac{\|u\|^{2}}{\left(\int_{\Omega}|u|^{6} d x\right)^{\frac{1}{3}}} .
$$

From [26], we know that $S$ is attained when $\Omega=R^{3}$ by functions

$$
y_{\varepsilon}=\frac{(3 \varepsilon)^{\frac{1}{4}}}{\left(\varepsilon+\left|x-x_{0}\right|^{2}\right)^{\frac{1}{2}}} .
$$

Definition A sequence $\left\{u_{n}\right\} \subset H_{0}^{1}(\Omega)$ is called a $(P S)_{c}$ sequence of $I$ if $I\left(u_{n}\right) \rightarrow c$ and $I^{\prime}\left(u_{n}\right) \rightarrow 0$ as $n \rightarrow \infty$. We say that $I$ satisfies the $(P S)_{c}$ condition if any $(P S)_{c}$ sequence $\left\{u_{n}\right\} \subset H_{0}^{1}(\Omega)$ of $I$ has a convergent subsequence.

Lemma 1 Let $a>0, b>0$ and $0<q<1$. Assume that $\left(f_{1}\right)$ and $\left(g_{1}\right)$ hold. If $\left\{u_{n}\right\} \subset H_{0}^{1}(\Omega)$ is $a(P S)_{c}$ sequence of $I$, then $\left\{u_{n}\right\}$ is bounded in $H_{0}^{1}(\Omega)$.

Proof By the Hölder inequality and the Young inequality, it follows from (3) and $\left(f_{1}\right)$ that

$$
\begin{aligned}
\left|\frac{\lambda}{q+1} \int_{\Omega} f\left(u^{+}\right)^{q+1} d x\right| & \leq \frac{\lambda}{q+1}\|f\|_{q_{0}}\|u\|_{6}^{q+1} \\
& \leq \frac{\lambda}{q+1} S^{-\frac{q+1}{2}}\|f\|_{q_{0}}\|u\|^{q+1} \\
& \leq \eta\|u\|^{2}+C(\eta) \lambda^{\frac{2}{1-q}}
\end{aligned}
$$

for any $u \in H_{0}^{1}(\Omega)$, where $C(\eta)=\left(\frac{\|f\|_{0}}{q+1}\right)^{\frac{2}{1-q}}(\eta S)^{\frac{q+1}{q-1}}$. Let $\left\{u_{n}\right\}$ be a $(P S)_{c}$ sequence of $I$. It follows from (4) that

$$
6 I\left(u_{n}\right)-\left\langle I^{\prime}\left(u_{n}\right), u_{n}\right\rangle \geq[2 a-(5-q) \eta]\left\|u_{n}\right\|^{2}-(5-q) C(\eta) \lambda^{\frac{2}{1-q}},
$$

which implies

$$
[2 a-(5-q) \eta]\left\|u_{n}\right\|^{2} \leq 6 c+(5-q) C(\eta) \lambda^{\frac{2}{1-q}}+o\left(\left\|u_{n}\right\|\right) .
$$

Set $\eta<\frac{2 a}{5-q}$, we see that $\left\{u_{n}\right\}$ is bounded in $H_{0}^{1}(\Omega)$.

Lemma 2 Let $a>0, b>0$, and $0<q<1$. Assume that $\left(f_{1}\right)$ and $\left(g_{1}\right)$ hold. If $\left\{u_{n}\right\}$ is a (PS)c sequence of $I$, then there exists a positive constant $A$ depending on $a, q, S$, and $\|f\|_{q_{0}}$ such that

$$
I^{\prime}(u)=0 \quad \text { and } \quad I(u) \geq-A \lambda^{\frac{2}{1-q}} .
$$


Proof Let $\left\{u_{n}\right\}$ be a $(P S)_{c}$ sequence of $I$. By Lemma 1, we know that $\left\{u_{n}\right\}$ is bounded. Therefore, up to a subsequence, there exists $u \in H_{0}^{1}(\Omega)$ such that $u_{n}$ converges weakly in $H_{0}^{1}(\Omega)$, strongly in $L^{s}(\Omega)$ with $1 \leq s<6$ and a.e. in $\Omega$. By $\left(f_{1}\right),\left(f_{2}\right)$, and the Dominated Convergence Theorem, we have

$$
\lambda \int_{\Omega} f\left(u_{n}^{+}\right)^{q}\left(u_{n}-u\right) d x+\int_{\Omega} g\left(u_{n}^{+}\right)^{5}\left(u_{n}-u\right) d x \rightarrow 0 .
$$

Thus, by using also the fact that $\left\langle I^{\prime}\left(u_{n}\right), u_{n}-u\right\rangle \rightarrow 0$, we get

$$
\left(a+b\left\|u_{n}\right\|^{2}\right) \int_{\Omega} \nabla u_{n} \cdot\left(\nabla u_{n}-\nabla u\right) d x \rightarrow 0
$$

from which it follows that $u_{n} \rightarrow u$ in $H_{0}^{1}(\Omega)$. Since $I$ is $C^{1}$, we obtain $I^{\prime}(u)=0$. In particular, we have $\left\langle I^{\prime}(u), u\right\rangle=0$, which implies that

$$
a\|u\|^{2}+b\|u\|^{4}=\lambda \int_{\Omega} f\left(u^{+}\right)^{q+1} d x+\int_{\Omega} g\left(u^{+}\right)^{6} d x .
$$

It follows from (4) that

$$
\begin{aligned}
I(u) & =I(u)-\frac{1}{6}\left\langle I^{\prime}(u), u\right\rangle \\
& =\frac{a}{3}\|u\|^{2}+\frac{b}{12}\|u\|^{4}-\frac{5-q}{6(q+1)} \lambda \int_{\Omega} f\left(u^{+}\right)^{q+1} d x \\
& \geq \frac{a}{3}\|u\|^{2}-\frac{5-q}{6}\left(\eta\|u\|^{2}+C(\eta) \lambda^{\frac{2}{1-q}}\right) .
\end{aligned}
$$

Set $\eta=\frac{2 a}{5-q}$, and we have $I(u) \geq-A \lambda^{\frac{2}{1-q}}$.

Lemma 3 Let $a>0, b>0$ and $0<q<1$. Assume that $\left(f_{1}\right)$ and $\left(g_{1}\right)$ hold, then I satisfies the $(P S)_{c}$ condition with $c<c^{*}=\frac{a b}{4}\|g\|_{\infty}^{-1} S^{3}+\frac{b^{3}}{24}\|g\|_{\infty}^{-2} S^{6}+\frac{a S}{6} \sqrt{b^{2}\|g\|_{\infty}^{-2} S^{4}+4 a\|g\|_{\infty}^{-1} S}+$ $\frac{b^{2}}{24}\|g\|_{\infty}^{-1} S^{4} \sqrt{b^{2}\|g\|_{\infty}^{-2} S^{4}+4 a\|g\|_{\infty}^{-1} S}-A \lambda^{\frac{2}{1-q}}$, where $A$ is the positive constant given in Lemma 2.

Proof Let $\left\{u_{n}\right\} \subset H_{0}^{1}(\Omega)$ be a $(P S)_{c}$ sequence of $I$ with $c<c^{*}$. By Lemma 1 , we know that $\left\{u_{n}\right\}$ is bounded. Up to a subsequence, we may assume that

$$
\begin{cases}u_{n} \rightarrow u, & \text { in } H_{0}^{1}(\Omega), \\ u_{n} \rightarrow u, & \text { a.e. on } \Omega, \\ u_{n} \rightarrow u, & \text { in } L^{s}(\Omega), 1 \leq s<6\end{cases}
$$

From Lemma 2, we have $I^{\prime}(u)=0$. By $\left(f_{1}\right)$ and the Dominated Convergence Theorem, we obtain

$$
\int_{\Omega} f\left(u_{n}^{+}\right)^{q+1} d x=\int_{\Omega} f\left(u^{+}\right)^{q+1} d x+o(1) .
$$

Let $w_{n}=u_{n}-u$; by the Brezis-Lieb lemma [27], one has

$$
\left\|u_{n}\right\|^{2}=\left\|w_{n}\right\|^{2}+\|u\|^{2}+o(1), \quad\left\|u_{n}\right\|^{4}=\left\|w_{n}\right\|^{4}+2\left\|w_{n}\right\|^{2}\|u\|^{2}+\|u\|^{4}+o(1)
$$


and

$$
\int_{\Omega} g\left(u_{n}^{+}\right)^{6} d x=\int_{\Omega} g\left(w_{n}^{+}\right)^{6} d x+\int_{\Omega} g\left(u^{+}\right)^{6} d x+o(1) .
$$

Since $I\left(u_{n}\right)=c+o(1)$, we obtain

$$
\frac{a}{2}\left\|w_{n}\right\|^{2}+\frac{b}{4}\left\|w_{n}\right\|^{4}+\frac{b}{2}\left\|w_{n}\right\|^{2}\|u\|^{2}-\frac{1}{6} \int_{\Omega} g\left(w_{n}^{+}\right)^{6} d x=c-I(u)+o(1) .
$$

According to $I^{\prime}\left(u_{n}\right)=o(1)$ and $\left\langle I^{\prime}(u), u\right\rangle=0$, we get

$$
a\left\|w_{n}\right\|^{2}+b\left\|w_{n}\right\|^{4}+b\left\|w_{n}\right\|^{2}\|u\|^{2}-\int_{\Omega} g\left(w_{n}^{+}\right)^{6} d x=o(1) .
$$

Assume that $\left\|w_{n}\right\| \rightarrow l$, it follows from (6) that

$$
\int_{\Omega} g\left(w_{n}^{+}\right)^{6} d x \rightarrow a l^{2}+b l^{4}+b l^{2}\|u\|^{2}
$$

From (3), we have

$$
\left\|w_{n}\right\|^{6} \geq S^{3} \int_{\Omega}\left|w_{n}\right|^{6} d x \geq S^{3}\|g\|_{\infty}^{-1} \int_{\Omega} g\left(w_{n}^{+}\right)^{6} d x .
$$

As $n \rightarrow \infty$, we deduce that

$$
l^{2} \geq \frac{b}{2}\|g\|_{\infty}^{-1} S^{3}+\frac{S}{2} \sqrt{b^{2}\|g\|_{\infty}^{-2} S^{4}+4\left(a+b\|u\|^{2}\right)\|g\|_{\infty}^{-1} S} .
$$

It follows from (5), (6), and Lemma 2 that

$$
\begin{aligned}
c= & \frac{a}{3} l^{2}+\frac{b}{12} l^{4}+\frac{b}{3} l^{2}\|u\|^{2}+I(u) \\
\geq & \frac{a}{3} l^{2}+\frac{b}{12} l^{4}-A \lambda^{\frac{2}{1-q}} \\
\geq & \frac{a b}{4}\|g\|_{\infty}^{-1} S^{3}+\frac{b^{3}}{24}\|g\|_{\infty}^{-2} S^{6}+\frac{a S}{6} \sqrt{b^{2}\|g\|_{\infty}^{-2} S^{4}+4\left(a+b\|u\|^{2}\right)\|g\|_{\infty}^{-1} S} \\
& +\frac{b^{2}}{24}\|g\|_{\infty}^{-1} S^{4} \sqrt{b^{2}\|g\|_{\infty}^{-2} S^{4}+4\left(a+b\|u\|^{2}\right)\|g\|_{\infty}^{-1} S}-A \lambda^{\frac{2}{1-q}} \\
\geq & c^{*}
\end{aligned}
$$

which contradicts the fact that $c<c^{*}$. Therefore, we have $l=0$, which implies that $u_{n} \rightarrow u$ in $H_{0}^{1}(\Omega)$. Hence $I$ satisfies the $(P S)_{c}$ condition with $c<c^{*}$.

\section{The proof of the main results}

In this section, we show the proofs of our Theorems 1 and 2. Before we come to the proof of Theorem 1, we first recall the following lemma in [28].

Lemma 4 Let $r, s>1, \psi \in L^{s}(\Omega)$ and $\psi^{+}=\max \{\psi, 0\} \neq 0$. Then there exists $w_{0} \in C_{0}^{\infty}(\Omega)$ such that $\int_{\Omega} \psi\left(w_{0}^{+}\right)^{r} d x>0$. 
Proof of Theorem 1 Using the hypotheses $\left(f_{1}\right)$ and $\left(g_{1}\right)$, it follows from (3) and (4) that

$$
I(u) \geq\left(\frac{a}{2}-\eta\right)\|u\|^{2}-C(\eta) \lambda^{\frac{2}{1-q}}-\frac{1}{6}\|g\|_{\infty} S^{-3}\|u\|^{6}
$$

Let $\eta=\frac{a}{4}$, we can find $\rho>0$ and $\Lambda_{1}>0$ such that for all $\lambda \in\left(0, \Lambda_{1}\right)$

$$
I(u)>0 \quad \text { if }\|u\|=\rho \quad \text { and } \quad I(u)>-C_{1} \quad \text { if }\|u\| \leq \rho
$$

where $C_{1}=\left(\frac{\|f\|_{q_{0}} \Lambda_{1}}{q+1}\right)^{\frac{2}{1-q}}\left(\frac{a}{4} S\right)^{\frac{q+1}{q-1}}$.

From Lemma 4 , we obtain the result that there exists $\varphi_{0} \in C_{0}^{\infty}(\Omega) \subset H_{0}^{1}(\Omega)$ such that

$$
\int_{\Omega} f\left(\varphi_{0}^{+}\right)^{q+1} d x>0
$$

Therefore, one has

$$
I\left(k \varphi_{0}\right) \leq \frac{a}{2} k^{2}\left\|\varphi_{0}\right\|^{2}+\frac{b}{4} k^{4}\left\|\varphi_{0}\right\|^{4}-\frac{\lambda}{q+1} k^{q+1} \int_{\Omega} f\left(\varphi_{0}^{+}\right)^{q+1} d x+\frac{1}{6} k^{6}\|g\|_{\infty}\left\|\varphi_{0}\right\|^{6} .
$$

Fix $\lambda \in\left(0, \Lambda_{1}\right)$; noticing that $0<q<1$, it implies from (8) that there exists $k_{0}=k(\lambda)>0$ small enough such that $I\left(k_{0} \varphi_{0}\right)<0$. Thus we deduce that

$$
c_{\lambda}=\inf _{u \in B_{\rho}(0)} I(u)<0<\inf _{u \in \partial B_{\rho}(0)} I(u) .
$$

By applying the Ekeland's variational principle in $\overline{B_{\rho}(0)}$ [29], we obtain the result that there exists a $(P S)_{c_{\lambda}}$ sequence $\left\{u_{n}\right\} \subset \overline{B_{\rho}(0)}$ of $I$.

By the expression of $c^{*}$, we can choose $0<\Lambda<\Lambda_{1}$ such that $c^{*}>0$ for all $\lambda \in(0, \Lambda)$. It follows from $c_{\lambda}<0$ and Lemma 3 that $I$ satisfies the $(P S)_{c_{\lambda}}$ condition. Therefore, one has a subsequence still denoted by $\left\{u_{n}\right\}$ and $u_{\lambda} \in H_{0}^{1}(\Omega)$ such that $u_{n} \rightarrow u_{\lambda}$ in $H_{0}^{1}(\Omega)$ and

$$
I\left(u_{\lambda}\right)=c_{\lambda}, \quad I^{\prime}\left(u_{\lambda}\right)=0
$$

which implies that $u_{\lambda}$ is a solution of problem (1). After a direct calculation, we derive $\left\|u_{\lambda}^{-}\right\|=\left\langle I^{\prime}\left(u_{\lambda}\right),-u_{\lambda}^{-}\right\rangle=0$, which implies $u_{\lambda} \geq 0$. Since $I\left(u_{\lambda}\right)=c_{\lambda}<0=I(0)$, we have $u_{\lambda} \neq 0$. Applying the Harnack inequality [30], we see that $u_{\lambda}$ is a positive solution of problem (1). The proof of Theorem 1 is completed.

Lemma 5 Let $a>0, b>0$ and $0<q<1$. Assume that $\left(f_{1}\right),\left(f_{2}\right),\left(g_{1}\right),\left(g_{2}\right)$, and $\left(g_{3}\right)$ hold, then there exists $\Lambda^{*}>0$, such that for any $\lambda \in\left(0, \Lambda^{*}\right)$, we can find $\bar{u}_{\lambda} \in H_{0}^{1}(\Omega)$ such that $\sup _{t \geq 0} I\left(t \bar{u}_{\lambda}\right)<c^{*}$.

Proof For convenience, we consider the functional $J: H_{0}^{1}(\Omega) \rightarrow R$ defined by

$$
J(u)=\frac{a}{2}\|u\|^{2}+\frac{b}{4}\|u\|^{4}-\frac{1}{6} \int_{\Omega} g\left(u^{+}\right)^{6} d x
$$

for all $u \in H_{0}^{1}(\Omega)$. According to $\left(g_{2}\right)$ and $\left(g_{3}\right)$, we can choose such a cut-off function $\phi(x) \in$ $C_{0}^{\infty}(\Omega)$ that $\phi(x)=1$ for $x \in B\left(x_{0}, \delta_{0}\right), \phi(x)=0$ for $x \in R^{3} \backslash B\left(x_{0}, 2 \delta_{0}\right), 0 \leq \phi(x) \leq 1$ and 
$|\nabla \phi| \leq C_{2}$, where $C_{2}>0$ is a positive constant. Define

$$
u_{\varepsilon}(x)=\frac{\phi(x)}{\left(\varepsilon+\left|x-x_{0}\right|^{2}\right)^{\frac{1}{2}}} .
$$

According to $\left(g_{2}\right)$ and $\left(g_{3}\right)$, similar to the calculation of [31], we have the following estimate $($ as $\varepsilon \rightarrow 0)$

$$
\begin{aligned}
& \left(\int_{\Omega} g\left|u_{\varepsilon}\right|^{6} d x\right)^{\frac{1}{3}}=\varepsilon^{-\frac{1}{2}}\|g\|_{\infty}^{\frac{1}{3}}|U|_{L^{6}\left(R^{3}\right)}^{2}+O(\varepsilon), \\
& \left\|u_{\varepsilon}\right\|^{2}=\varepsilon^{-\frac{1}{2}}|\nabla U|_{L^{2}\left(R^{3}\right)}^{2}+O(1), \\
& \frac{\left\|u_{\varepsilon}\right\|^{2}}{\left(\int_{\Omega} g\left|u_{\varepsilon}\right|^{6} d x\right)^{\frac{1}{3}}}=\|g\|_{\infty}^{-\frac{1}{3}} S+O\left(\varepsilon^{\frac{1}{2}}\right),
\end{aligned}
$$

where $U(x)=\left(1+\left|x-x_{0}\right|^{2}\right)^{-\frac{1}{2}}$. Define

$$
h(t)=J\left(t u_{\varepsilon}\right)=\frac{a}{2} t^{2}\left\|u_{\varepsilon}\right\|^{2}+\frac{b}{4} t^{4}\left\|u_{\varepsilon}\right\|^{4}-\frac{1}{6} t^{6} \int_{\Omega} g\left|u_{\varepsilon}\right|^{6} d x
$$

for all $t \geq 0$. From (9), we have $\lim _{t \rightarrow+\infty} h(t)=-\infty$. Note that $h(0)=0$ and $h(t)>0$ for $t \rightarrow 0^{+}$, so $\sup _{t \geq 0} h(t)$ is attained for some $t_{\varepsilon}>0$. By

$$
0=h^{\prime}\left(t_{\varepsilon}\right)=t_{\varepsilon}\left(a\left\|u_{\varepsilon}\right\|^{2}+b t_{\varepsilon}^{2}\left\|u_{\varepsilon}\right\|^{4}-t_{\varepsilon}^{4} \int_{\Omega} g\left|u_{\varepsilon}\right|^{6} d x\right),
$$

one has

$$
t_{\varepsilon}^{2}=\frac{b\left\|u_{\varepsilon}\right\|^{4}+\sqrt{b^{2}\left\|u_{\varepsilon}\right\|^{8}+4 a\left\|u_{\varepsilon}\right\|^{2} \int_{\Omega} g\left|u_{\varepsilon}\right|^{6} d x}}{2 \int_{\Omega} g\left|u_{\varepsilon}\right|^{6} d x} .
$$

Therefore, we deduce from (9) that

$$
\begin{aligned}
\sup _{t \geq 0} J\left(t u_{\varepsilon}\right)= & \frac{a b\left\|u_{\varepsilon}\right\|^{6}}{4 \int_{\Omega} g\left|u_{\varepsilon}\right|^{6} d x}+\frac{a \sqrt{b^{2}\left\|u_{\varepsilon}\right\|^{12}+4 a\left\|u_{\varepsilon}\right\|^{6} \int_{\Omega} g\left|u_{\varepsilon}\right|^{6} d x}}{6 \int_{\Omega} g\left|u_{\varepsilon}\right|^{6} d x} \\
& +\frac{b^{3}\left\|u_{\varepsilon}\right\|^{12}}{24\left(\int_{\Omega} g\left|u_{\varepsilon}\right|^{6} d x\right)^{2}}+\frac{b^{2} \sqrt{b^{2}\left\|u_{\varepsilon}\right\|^{24}+4 a\left\|u_{\varepsilon}\right\|^{18} \int_{\Omega} g\left|u_{\varepsilon}\right|^{6} d x}}{24\left(\int_{\Omega} g\left|u_{\varepsilon}\right|^{6} d x\right)^{2}} \\
= & \frac{a b}{4}\|g\|_{\infty}^{-1} S^{3}+O\left(\varepsilon^{\frac{1}{2}}\right)+\frac{b^{3}}{24}\|g\|_{\infty}^{-2} S^{6}+O\left(\varepsilon^{\frac{1}{2}}\right) \\
& +\frac{a S}{6} \sqrt{b^{2}\|g\|_{\infty}^{-2} S^{4}+4 a\|g\|_{\infty}^{-1} S}+O\left(\varepsilon^{\frac{1}{4}}\right) \\
& +\frac{b^{2}}{24}\|g\|_{\infty}^{-1} S^{4} \sqrt{b^{2}\|g\|_{\infty}^{-2} S^{4}+4 a\|g\|_{\infty}^{-1} S}+O\left(\varepsilon^{\frac{1}{4}}\right) \\
= & c^{*}+A \lambda^{\frac{2}{1-q}}+O\left(\varepsilon^{\frac{1}{4}}\right) .
\end{aligned}
$$

By the expression of $c^{*}$, we can choose $\Lambda_{2}>0$ such that $c^{*}>0$ for all $\lambda \in\left(0, \Lambda_{2}\right)$. Using the definitions of $I$ and $u_{\varepsilon}$, from $\left(f_{2}\right)$ and $\left(g_{2}\right)$, we have

$$
I\left(t u_{\varepsilon}\right) \leq \frac{a}{2} t^{2}\left\|u_{\varepsilon}\right\|^{2}+\frac{b}{4} t^{4}\left\|u_{\varepsilon}\right\|^{4}
$$


for all $t \geq 0$ and $\lambda>0$. It follows that there exist $T \in(0,1)$ and $\varepsilon_{1}>0$ such that

$$
\sup _{0 \leq t \leq T} I\left(t u_{\varepsilon}\right) \leq c^{*}
$$

for all $0<\lambda<\Lambda_{2}$ and $0<\varepsilon<\varepsilon_{1}$. Moreover, using the definitions of $I$ and $u_{\varepsilon}$, it follows from $\left(f_{2}\right)$ and $(10)$ that

$$
\begin{aligned}
\sup _{t \geq T} I\left(t u_{\varepsilon}\right) & =\sup _{t \geq T}\left(J\left(t u_{\varepsilon}\right)-\frac{\lambda}{q+1} t^{q+1} \int_{\Omega} f\left|u_{\varepsilon}\right|^{q+1} d x\right) \\
& \leq c^{*}+A \lambda^{\frac{2}{1-q}}+O\left(\varepsilon^{\frac{1}{4}}\right)-\frac{\lambda \beta_{0}}{q+1} T^{q+1} \int_{B\left(x_{0}, \delta_{0}\right)}\left|u_{\varepsilon}\right|^{q+1} d x .
\end{aligned}
$$

Let $\varepsilon=\lambda^{\frac{8}{1-q}} \in\left(0, \delta_{0}^{2}\right)$, it follows that

$$
\begin{aligned}
\int_{B\left(x_{0}, \delta_{0}\right)}\left|u_{\varepsilon}\right|^{q+1} d x & =\int_{B\left(x_{0}, \delta_{0}\right)} \frac{1}{\left(\varepsilon+\left|x-x_{0}\right|^{2}\right)^{\frac{q+1}{2}}} d x \\
& \geq \int_{B\left(x_{0}, \delta_{0}\right)} \frac{1}{\left(2 \delta_{0}^{2}\right)^{\frac{q+1}{2}}} d x \\
& =C_{3} .
\end{aligned}
$$

By the above two inequalities, for any $0<\lambda<\delta_{0}^{\frac{1-q}{4}}$, we have

$$
\sup _{t \geq T} I\left(t u_{\varepsilon}\right) \leq c^{*}+O\left(\lambda^{\frac{2}{1-q}}\right)-\frac{C_{3} \beta_{0} \lambda}{q+1} T^{q+1} .
$$

Hence, we can choose $\Lambda_{3}>0$ such that for all $0<\lambda<\Lambda_{3}$

$$
O\left(\lambda^{\frac{2}{1-q}}\right)-\frac{C_{3} \beta_{0} \lambda}{q+1} T^{q+1}<0
$$

Therefore, for all $0<\lambda<\Lambda_{3}$ and $\varepsilon=\lambda^{\frac{8}{1-q}}$, we have

$$
\sup _{t \geq T} I\left(t u_{\varepsilon}\right) \leq c^{*}
$$

Set $\Lambda^{*}=\min \left\{\Lambda_{2}, \Lambda_{3}, \varepsilon_{1}^{\frac{1-q}{8}}\right\}$. Let $\lambda \in\left(0, \Lambda^{*}\right), \varepsilon=\lambda^{\frac{8}{1-q}}$ and $\bar{u}_{\lambda}=u_{\varepsilon}$, we deduce from (11) and (13) that

$$
\sup _{t \geq 0} I\left(t \bar{u}_{\lambda}\right)<c^{*}
$$

Proof of Theorem 2 Choose $\lambda^{*}=\min \left\{\Lambda, \Lambda^{*}\right\}$, from the proof of Theorem 1, we have already seen that problem (1) for any $\lambda \in\left(0, \lambda^{*}\right)$ has a positive solution $u_{\lambda}$ with $I\left(u_{\lambda}\right)<0$. Now we only need to find the second positive solution of problem (1). According to $\left(f_{1}\right)$, we can see that (4) and (7) hold. It follows from $\left(g_{1}\right)$ and Lemma 4 that there exists $\phi_{0} \in C_{0}^{\infty}(\Omega)$ such that

$$
\int_{\Omega} g\left(\phi_{0}^{+}\right)^{6} d x>0
$$


According to (4), we have

$$
I\left(t \phi_{0}\right) \leq\left(\frac{a}{2}+\eta\right) t^{2}\left\|\phi_{0}\right\|^{2}+\frac{1}{4} t^{4}\left\|\phi_{0}\right\|^{4}-\frac{1}{6} t^{6} \int_{\Omega} g\left(\phi_{0}^{+}\right)^{6} d x+C(\eta) \lambda^{\frac{2}{1-q}}
$$

which implies that

$$
I\left(t \phi_{0}\right) \rightarrow-\infty \quad \text { as } t \rightarrow+\infty
$$

Hence, there exists a positive number $t_{0}$ such that $\left\|t_{0} \phi_{0}\right\|>\rho$ and $I\left(t_{0} \phi_{0}\right)<0$ for any $\lambda \in$ $\left(0, \lambda^{*}\right)$. It implies from (7) that the functional $I$ has the mountain pass geometry. Define

$$
\Gamma=\left\{\gamma \in C\left([0,1], H_{0}^{1}(\Omega)\right) \mid \gamma(0)=0, \gamma(1)=t_{0} \phi_{0}\right\}, \quad \tilde{c}_{\lambda}=\inf _{\gamma \in \Gamma} \max _{t \in[0,1]} I(\gamma(t))
$$

From Lemma 5, we have $\tilde{c}_{\lambda}<c^{*}$. Applying Lemma 3, we know that $I$ satisfies the $(P S)_{\tilde{c}_{\lambda}}$ condition. By the Mountain Pass Theorem [32], we obtain the result that problem (1) has the second solution $\tilde{u}_{\lambda}$ with $I\left(\tilde{u}_{\lambda}\right)>0$. After a direct calculation, we derive

$$
\left\|\tilde{u}_{\lambda}^{-}\right\|^{2}=\left\langle I^{\prime}\left(\tilde{u}_{\lambda}\right),-\tilde{u}_{\lambda}^{-}\right\rangle=0,
$$

which implies that $\tilde{u}_{\lambda}^{-}=0$. Hence we have $\tilde{u}_{\lambda} \geq 0$. Since $I\left(\tilde{u}_{\lambda}\right)>0=I(0)$, we have $\tilde{u}_{\lambda} \neq 0$. By the Harnack inequality, we obtain the result that $\tilde{u}_{\lambda}$ is the second positive solution of problem (1). The proof of Theorem 2 is completed.

\section{Competing interests}

The author declares that they have no competing interests.

\section{Acknowledgements}

The author would like to thank the referees for valuable comments and suggestions on improving this paper. This paper was supported by Science and Education Youth culture project in Guizhou Province (Contract Number: Guizhou Provincial Institute of Zi (2012) No. 157). This paper also was supported by Science and Technology Foundation of Guizhou Province (No. J[2013]2141; No. LKM[2011]31)

Received: 29 August 2013 Accepted: 25 December 2013 Published: 16 Jan 2014

\section{References}

1. Kirchhoff, G: Mechanik. Teubner, Leipzig (1883)

2. Lions, JL: On some questions in boundary value problems of mathematical physics. In: Contemporary Developments in Continuum Mechanics and Partial Differential Equations (Proc. Internat. Sympos., Inst. Mat., Univ. Fed. Rio de Janeiro, Rio de Janeiro, 1977). North-Holland Math. Stud., vol. 30, pp. 284-346. North-Holland, Amsterdam (1978)

3. Ma, TF, Munoz Rivera, JE: Positive solutions for a nonlinear elliptic transmission problem. Appl. Math. Lett. 16(2), 243-248 (2003)

4. Alves, CO, Corra, FJSA, Ma, TF: Positive solutions for a quasilinear elliptic equation of Kirchhoff type. Comput. Math Appl. 49(1), 85-93 (2005)

5. Bensedki, A, Bouchekif, M: On an elliptic equation of Kirchhoff-type with a potential asymptotically linear at infinity. Math. Comput. Model. 49, 1089-1096 (2009)

6. Perera, K, Zhang, ZT: Nontrivial solutions of Kirchhoff-type problems via the Yang index. J. Differ. Equ. 221, 246-255 (2006)

7. He, XM, Zou, WM: Infinitely many positive solutions for Kirchhoff-type problems. Nonlinear Anal. 70(3), 1407-1414 (2009)

8. Figueiredo, GM: Existence of positive solution for a Kirchhoff problem type with critical growth via truncation argument. J. Math. Anal. Appl. 401, 706-713 (2013)

9. Liang, SH, Shi, SY: Soliton solutions to Kirchhoff type problems involving the critical growth in $R^{N}$. Nonlinear Anal. 81, 31-41 (2013)

10. Wang, J, Tian, LX, Xu, JX, Zhang, FB: Multiplicity and concentration of positive solutions for a Kirchhoff type problem with critical growth. J. Differ. Equ. 253(7), 2314-2351 (2012)

11. Sun, YJ, Liu, X: Existence of positive solutions for Kirchhoff type problems with critical exponent. J. Partial Differ. Equ. 25(2), 187-198 (2012) 
12. Alves, CO, Correa, FJSA, Figueiredo, GM: On a class of nonlocal elliptic problems with critical growth. Differ. Equ. Appl. 2(3), 409-417 (2010)

13. Ahmed, H, Mohammed, M, Najib, T: Existence of solutions for $p$-Kirchhoff type problems with critical exponent. Electron. J. Differ. Equ. 2011, 105 (2011)

14. Chen, CY, Kuo, YC, Wu, TF: The Nehari manifold for a Kirchhoff type problem involving sign-changing weight functions. J. Differ. Equ. 250, 1876-1908 (2011)

15. Li, YH, Li, FY, Shi, JP: Existence of a positive solution to Kirchhoff type problems without compactness conditions. J. Differ. Equ. 253(7), 2285-2294 (2012)

16. He, XM, Zou, WM: Existence and concentration behavior of positive solutions for a Kirchhoff equation in $R^{3}$. J. Differ. Equ. 252(2), 1813-1834 (2012)

17. Chen, CS, Huang, JC, Liu, LH: Multiple solutions to the nonhomogeneous p-Kirchhoff elliptic equation with concave-convex nonlinearities. Appl. Math. Lett. 26(7), 754-759 (2013)

18. Cheng, BT, Wu, X, Liu, J: Multiple solutions for a class of Kirchhoff type problems with concave nonlinearity. Nonlinear Differ. Equ. Appl. 19(5), 521-537 (2012)

19. Azzouz, N, Bensedik, A: Existence results for an elliptic equation of Kirchhoff type with changing sign data. Funkc. Ekvacioj 55(1), 55-66 (2012)

20. Sun, J, Liu, SB: Nontrivial solutions of Kirchhoff type problems. Appl. Math. Lett. 25(3), 500-504 (2012)

21. Aouaoui, S: Existence of three solutions for some equation of Kirchhoff type involving variable exponents. Appl. Math. Comput. 218(13), 7184-7192 (2012)

22. Liu, W, He, XM: Multiplicity of high energy solutions for superlinear Kirchhoff equations. J. Appl. Math. Comput. 39(1-2), 473-487 (2012)

23. Liu, DC, Zhao, PH: Multiple nontrivial solutions to a p-Kirchhoff equation. Nonlinear Anal. 75(13), $5032-5038$ (2012)

24. Sun, JJ, Tang, CL: Existence and multiplicity of solutions for Kirchhoff type equations. Nonlinear Anal. 74(4), $1212-1222$ (2011)

25. Yang, Y, Zhang, JH: Positive and negative solutions of a class of nonlocal problems. Nonlinear Anal. 73(1), 25-30 (2010)

26. Talenti, G: Best constant in Sobolev inequality. Ann. Mat. Pura Appl. 110(4), 353-372 (1976)

27. Brézis, H, Lieb, E: A relation between pointwise convergence of functions and convergence of functional. Proc. Am. Math. Soc. 88, 486-490 (1983)

28. Chu, CM, Tang, CL: Multiple results for critical quasilinear elliptic systems involving concave-convex nonlinearities and sign-changing weight functions. Bull. Malays. Math. Soc. 36(3), 789-805 (2013)

29. Ekeland, I: On the variational principle. J. Math. Anal. Appl. 47, 324-353 (1974)

30. Trudinger, NS: On Harnack type inequalities and their application to quasilinear elliptic equations. Commun. Pure Appl. Math. 20, 721-747 (1967)

31. Hsu, TS, Lin, HL: Multiple positive solutions for singular elliptic equations with concave-convex nonlinearities and sign-changing weights. Bound. Value Probl. 2009, Article ID 584203 (2009)

32. Ambrosetti, A, Rabinowitz, PH: Dual variational methods in critical point theory and applications. J. Funct. Anal. 14, 347-381 (1973)

10.1186/1687-2770-2014-19

Cite this article as: Chu: Multiplicity of positive solutions for Kirchhoff type problem involving critical exponent and sign-changing weight functions. Boundary Value Problems 2014, 2014:19

\section{Submit your manuscript to a SpringerOpen ${ }^{\circ}$ journal and benefit from:}

- Convenient online submission

- Rigorous peer review

- Immediate publication on acceptance

Open access: articles freely available online

- High visibility within the field

- Retaining the copyright to your article 